Selcuk Journal of Agriculture and Food Sciences

http://sjafs.selcuk.edu.tr/sjafs/index

Research Article
SJAFS

(2020) 34 (1), 78-83

e-ISSN: $2458-8377$

DOI:10.15316/SJAFS.2020.198

\title{
The Antibacterial Effects of the Different Extracts of Oenothera biennis and Origanum minutiflorum O. Schwarz et. P. H. Davis on Food-borne Pathogenic Bacteria
}

\author{
Gökhan AKARCA ${ }^{1 *}$ Oktay TOMAR ${ }^{2}$ \\ ${ }^{1}$ Afyon Kocatepe University, Faculty of Engineering, Department of Food Engineering, Afyonkarahisar, Turkey \\ ${ }^{2}$ Kocaeli University, Faculty of Agriculture and Natural Science, Arslanbey Campus, Kocaeli, Turkey
}

\begin{tabular}{l}
\hline ARTICLE INFO \\
\hline Article history: \\
Received date: 20.12 .2019 \\
Accepted date: 14.02 .2020 \\
\hline Edited by: \\
Cemalettin SARIÇOBAN; Selçuk Uni- \\
versity, Turkey \\
Reviewed by: \\
Yavuz YÜKSEL; Afyon Kocatepe Uni- \\
versity, Turkey \\
Hasan Hüseyin KARA; Necmettin Erba- \\
kan University, Turkey \\
\hline Keywords: \\
Oenothera biennis \\
Origanum minutiflorum O. Schwarz et. \\
P. H. Davis \\
Extract \\
Antibacterial Effect \\
Escherichia coli \\
Listeria monocytogenes
\end{tabular}

\section{Introduction}

Oenothera biennis is a biennial plant from the Convolvulaceae family. It is commonly known as evening primrose. The plant grows well in sandy, loamy and clayey soils in almost every place such as fields, roadsides and meadows (Morrison and Reekie, 1995). The oil obtained from the seeds of the plant was determined to have various pharmacological properties (Arimura, 2003a). The seed oil of the plant is very rich in $\gamma$ linolenic acid content, an important fatty acid found in the composition of prostaglandins and related hormones that are effective in regulating muscle and vascular contractions in human physiology. (Becker, 1983; Shukla et al., 1999). Also, recent studies have shown that ethanol extract obtained from the seeds of the plant shows specific anti-tumor activity (Arimura et al., 2003b; Arimura et al., 2004).

\footnotetext{
*Corresponding author email: gakarca@aku.edu.tr
}

The Origanum genus belonging to the Lamiaceae family has 24 species in Turkey. Of these species, 16 are endemic (Aslım and Yücel, 2008; Albayrak and Aksoy, 2017). They are widely grown in the Mediterranean region (Azizi et al., 2009; Oke and Aslim, 2010). The species has high-level biological properties and reported to possess antimicrobial, antifungal, antioxidant, antimutagenic, anticarcinogenic, antifungal, antinematodal, antiparasitic and antiemetic activities (Bostancioglu et al., 2012; Chishti et al., 2013; Karaboduk et al., 2014; Sarikurkcu et al., 2015). Origanum minutiflorum O. Schwarz et. P. H. Davis (Turkish oregano) is an endemic species that grows in the Sütçüler region of Isparta in Turkey (Baydar, 2005). It is widely used as a spice and herbal tea (Ozen et al., 2014).

This study aimed to determine the antibacterial effects of the extracts obtained from the leaves of Oenothera biennis and Origanum minutiflorum O. Schwarz et. P. H. on foodborne pathogenic bacteria. 


\section{Materials and Methods}

\subsection{Materials}

Oenothera biennis used in the study was obtained from the villages in Antalya while Origanum minutiflorum O. Schwarz et. P. H. Davis was obtained from the Sütçüler region in Isparta, Turkey.

\subsection{Bacterial Strains Used in This Study}

In the study; Staphylococcus aureus (ATCC 6538), Yersinia enterocolitica (ATCC 9610), Salmonella Typhimurium (ATCC 14028), Listeria monocytogenes (ATCC 51774), Escherichia coli (ATCC 25922) Enterococcus feacalis (ATCC 29212), Enterobacter aerogenes (ATCC 13048) ve Shigella flexneri (ATCC 12022) Bacillus cereus (ATCC 14579) Pseudomonas aeroginosa (ATCC 15442), Escherichia coli (ATCC 25922) species of bacteria were used.

\subsection{Preparation of Plant Extracts}

Leaves of the plants were cut into small pieces and mixed with $400 \mathrm{~mL}$ ethanol (85\%: Merck, 100983, Germany), methanol (Merck, 106009 Germany), diethyl ether (Merck, 100921, Germany), acetone (Merck, 100014, Germany) or chloroform (Merck, 102445, Germany) at 1:3 (w/v) ratio. The mixtures were then shaken at $22{ }^{\circ} \mathrm{C}$ in a shaker (Wiseshake SHO-2D, Witeg, Germany) at $120 \mathrm{rpm}$ for 24 hours. After the extracts were filtered through sterilized filter paper (Whatman No. 32), the solvents were removed from the extract by rotary evaporator (Heidolph, Germany). The extracts were stored in colored glass bottles (100 ml, glass bottle, Turkey) at $4^{\circ} \mathrm{C}$ in refrigerator (Arçelik 554271, Turkey).

\subsection{Preparation of Discs Containing Plant Extracts}

For the preparation of discs containing the plant extracts, $10 \mu \mathrm{L}$ samples of the extracts of Oenothera biennis and Origanum minutiflorum O. Schwarz et. P. H. Davis were taken into Petri dishes (Sterile, $90 \times 15$, Firatmed, Turkey) using sterile tipped pipettes (Research Plus, Eppendorf) and dropped on 6-mmdiameter empty antibiogram discs (Bio-Disk 316010001). The Petri dishes were kept closed at $4{ }^{\circ} \mathrm{C}$ in refrigerator (Arçelik 554271, Turkey) for 60 minutes for the discs to absorb the extracts. The extractimpregnated discs were then dried in a laminar flow cabinet (Cryste, Puricube 1200) at room temperature for 8-10 hours.

\subsection{Preparation of the Inocula}

The young (24-hour) bacterial strains produced on non-selective media were taken from single growing colonies using a sterile loop and suspended in physiological saline (Merck, 115525, Germany) until homogeneous turbidity occurred. The density of the inoculum suspension was adjusted to $0.5 \mathrm{McFarland}$ standard using a densitometer (Biosen, 1B, Turkey). The inocula were taken using a transport swap (Firatmed, Turkey) and inoculated on the surface of Mueller Hin- ton Agar (1.05437, Merck, Germany) (MHA) and spread homogeneously (Bauer et al., 1966; Akarca, 2019).

After waiting for 10 minutes for the medium to absorb the inocula, the antibiogram discs containing plant extracts were placed in the Petri dishes and incubated in an incubator (Incucell, MMM, Germany) as described by Anonymous (2018) and Cruz-Gálvez et al. (2018). The zones formed at the end of the period were measured in mm using a digital caliper (Mitutoyo, 500181-30, Japan) under sufficient daylight.

\subsection{Minimum Inhibitory Concentration (MIC) and Minimum Bactericidal Concentration (MBC) Values}

From the extracts of both plants obtained with five different solvents, $1 \mathrm{~mL}$ of Nutrient Broth (Merck, 1.05443 , Germany) was added to the first tubes. Then, $1 \mathrm{~mL}$ of the mixtures formed in the first tubes was taken and transferred to the next tubes. This procedure was applied to all tubes in series. As a result, the mixtures were obtained in equal amounts in each tube but only half of the concentration of the previous tube. Also, positive and negative control tubes were formed.

Of the bacterial strains used in the study, $1 \mu \mathrm{l}\left(10^{6}\right.$ $\mathrm{cfu} / \mathrm{mL}$ ) were inoculated into all other tubes except for the negative control tube and incubated at the appropriate temperature, time and conditions. At the end of the period, turbidity in the tubes, membrane formation on the surface and sediment at the bottom were regarded as positive. Also, no growth was determined in the negative control tube whereas growth was determined in the positive control tube. The MIC value was determined by taking half of the sum of the concentrations of the first tube evaluated as positive growth and the tubes previously evaluated as negative growth (By Aamer et al., 2015; Chikezie, 2017; Akarca, 2019).

The first tube, which was evaluated as negative growth in MIC analysis, was inoculated into Muller Hinton Agar (Merck, 195437, Germany) by taking $1 \mu \mathrm{l}$ from all the tubes at the following concentrations and then incubated at the appropriate temperature, time and conditions for each bacterial species. The value of the first concentration with no growth at the end of the period was evaluated as MBC (Dhiman et al., 2011; By Aamer et al., 2015; Akarca, 2019).

\subsection{Statistical Analysis}

The results of the study were determined by SPSS (V 23.0.0) statistical software and the differences were determined by the Duncan test $(\mathrm{P}<0.05)$.

\section{Results and Discussion}

The antibacterial effect (mm-zone-diameter) of the extracts of Oenothera biennis from five different solvents on ten foodborne pathogenic bacteria is shown in Table 1.

It was determined that the diethyl ether extract of Oenothera biennis showed the highest antibacterial 
effect on Listeria monocytogenes with a 37.23-mmzone-diameter, followed by Bacillus cereus with a 32.46-mm-zone diameter $(\mathrm{P}<0.05)$. In contrast, the acetone extract exhibited the lowest antibacterial effect $(\mathrm{P}<0.05)$. Pseudomonas aeroginosa was the bacteria species on which this extract showed the lowest anti- bacterial effect with a 7.33-mm-zone-diameter $(\mathrm{P}<0.05)$.

It was determined that the highest antibacterial effect was determined in the Origanum minutiflorum $\mathrm{O}$. Schwarz et. P. H. extract obtained using diethyl ether, followed by extracts obtained using ethanol and chloroform (Table $2 ; \mathrm{P}<0.05$ ).

Table 1

Antibacterial Effects of Different Extracts of Oenothera biennis (mm Zone Diameter)

\begin{tabular}{|c|c|c|c|c|c|}
\hline \multirow{2}{*}{ Species of Bacteria } & \multicolumn{5}{|c|}{ Solvent } \\
\hline & Ethanol & Methanol & Diethyl ether & Acetone & Chloroform \\
\hline Staphylococcus aureus & $16.02 \pm 1.39^{\mathrm{Bde}}$ & $15.08 \pm 0.65^{\mathrm{Bbc}}$ & $20.55 \pm 0.93^{\mathrm{Ad}}$ & $9.93 \pm 0.25^{\mathrm{Cbc}}$ & $14.28 \pm 1.02^{\mathrm{Bcd}}$ \\
\hline Yersinia enterecolitica & $18.00 \pm 1.57^{\mathrm{Ad}}$ & $10.29 \pm 0.96^{\mathrm{Bef}}$ & $18.18 \pm 0.54^{\text {Aef }}$ & $12.29 \pm 1.14^{\mathrm{Bab}}$ & $13.79 \pm 1.66^{\mathrm{ABcd}}$ \\
\hline Salmonella Typhimurium & $21.85 \pm 1.24^{\mathrm{Ac}}$ & $14.61 \pm 1.12^{\mathrm{Bbc}}$ & $23.18 \pm 0.34^{\mathrm{Ac}}$ & $13.80 \pm 2.67^{\mathrm{Ba}}$ & $15.87 \pm 0.51^{\mathrm{Bbc}}$ \\
\hline Listeria monocytogenes & $35.81 \pm 0.62^{\mathrm{Aa}}$ & $16.76 \pm 1.39^{\mathrm{Bb}}$ & $37.23 \pm 0.90^{\mathrm{Aa}}$ & $15.15 \pm 1.03^{\mathrm{Ba}}$ & $17.30 \pm 0.88^{\mathrm{Bb}}$ \\
\hline Enterococcus feacalis & $17.86 \pm 0.38^{\mathrm{Ad}}$ & $12.01 \pm 0.14^{\text {Bde }}$ & $18.43 \pm 0.66^{\text {Aef }}$ & $9.35 \pm 0.67^{\mathrm{Cbc}}$ & $10.22 \pm 0.30^{\mathrm{Cf}}$ \\
\hline Enterobacter aerogenes & $14.77 \pm 0.40^{\text {Ade }}$ & $10.02 \pm 0.51^{\mathrm{Bef}}$ & $16.25 \pm 0.11^{\mathrm{Af}}$ & $9.16 \pm 0.44^{\mathrm{Bbc}}$ & $10.71 \pm 0.53^{\mathrm{Bf}}$ \\
\hline Shigella flexneri & $16.96 \pm 1.28^{\mathrm{ABde}}$ & $13.77 \pm 0.58^{\mathrm{Bcd}}$ & $17.24 \pm 0.91^{\mathrm{Aef}}$ & $10.08 \pm 0.07^{\mathrm{Cbc}}$ & $13.94 \pm 1.07^{\mathrm{ABcd}}$ \\
\hline Bacillus cereus & $30.65 \pm 1.70^{\mathrm{Ab}}$ & $19.28 \pm 0.80^{\mathrm{Ba}}$ & $32.46 \pm 0.71^{\mathrm{Ab}}$ & $15.28 \pm 1.11^{\mathrm{Ca}}$ & $20.36 \pm 0.78^{\mathrm{Ba}}$ \\
\hline Pseudomonas aeroginosa & $13.36 \pm 0.82^{\mathrm{Be}}$ & $8.86 \pm 0.22^{\mathrm{Cf}}$ & $19.23 \pm 1.12^{\mathrm{Ade}}$ & $7.33 \pm 0.21^{\mathrm{Cc}}$ & $9.04 \pm 0.23^{\mathrm{Cf}}$ \\
\hline Escherichia coli & $17.05 \pm 1.34^{\mathrm{ABde}}$ & $9.32 \pm 0.16^{\mathrm{Cf}}$ & $20.12 \pm 1.37^{\mathrm{Ad}}$ & $15.19 \pm 0.96^{\mathrm{Ba}}$ & $11.27 \pm 1.10^{\mathrm{Cde}}$ \\
\hline
\end{tabular}

a-f $(\downarrow)$ : Values with the same capital letters in the same column for each analysis differ significantly $(\mathrm{P}<0.05)$

A-C $(\rightarrow)$ : Values with the same capital letters in the same rows for each analysis differ significantly $(\mathrm{P}<0.05)$.

Table 2

Antibacterial Effects of Different Extracts of Origanum minutiflorum O. Schwarz et. P. H. (mm Zone Diameter)

\begin{tabular}{|c|c|c|c|c|c|}
\hline \multirow{2}{*}{ Species of Bacteria } & \multicolumn{5}{|c|}{ Solvent } \\
\hline & Ethanol & Methanol & Diethyl Ether & Acetone & Chloroform \\
\hline Staphylococcus aureus & $13.88 \pm 0.64^{\mathrm{Bb}}$ & $10.99 \pm 0.25^{\mathrm{Cb}}$ & $16.30 \pm 0.83^{\mathrm{Ac}}$ & $10.72 \pm 0.36^{\mathrm{Cc}}$ & $11.33 \pm 0.21^{\mathrm{Cbc}}$ \\
\hline Yersinia enterecolitica & $11.09 \pm 0.23^{\mathrm{B}}$ & $10.01 \pm 0.36^{\mathrm{Bb}}$ & $12.84 \pm 0.32^{\text {Aef }}$ & $9.96 \pm 0.09^{\mathrm{Bc}} \mathrm{d}$ & $10.63 \pm 0.50^{\mathrm{Bcd}}$ \\
\hline Salmonella Typhimurium & $12.84 \pm 0.39^{\mathrm{ABbcd}}$ & $10.65 \pm 0.97^{\mathrm{BCb}}$ & $13.35 \pm 0.86^{\mathrm{Ad}}$ & $10.25 \pm 0.47^{\mathrm{Ccd}}$ & $12.07 \pm 0.19^{\mathrm{ABCb}}$ \\
\hline Listeria monocytogenes & $20.86 \pm 0.32^{\mathrm{Ba}}$ & $15.55 \pm 0.93^{\mathrm{Ca}}$ & $23.98 \pm 0.27^{\mathrm{Aa}}$ & $13.14 \pm 0.52^{\mathrm{Db}}$ & $16.77 \pm 0.46^{\mathrm{Ca}}$ \\
\hline Enterococcus feacalis & $10.61 \pm 0.75^{\mathrm{Ae}}$ & $9.45 \pm 0.71^{\mathrm{ABb}}$ & $10.90 \pm 0.53^{\mathrm{Af}}$ & $8.13 \pm 0.44^{\mathrm{Bf}}$ & $10.13 \pm 0.21^{\mathrm{ABcd}}$ \\
\hline Enterobacter aerogenes & $11.00 \pm 0.33^{\mathrm{Ade}}$ & $9.77 \pm 0.50^{\mathrm{Ab}}$ & $11.56 \pm 1.16^{\text {Aef }}$ & $9.33 \pm 0.30^{\text {Ade }}$ & $10.45 \pm 0.12^{\text {Acd }}$ \\
\hline Shigella flexneri & $10.97 \pm 0.35^{\text {Bde }}$ & $9.92 \pm 0.37^{\mathrm{Bb}}$ & $13.23 \pm 0.41^{\mathrm{Aef}}$ & $9.84 \pm 0.29^{\mathrm{Bcd}}$ & $9.78 \pm 0.33^{\mathrm{Bd}}$ \\
\hline Bacillus cereus & $19.42 \pm 1.04^{\mathrm{ABa}}$ & $16.10 \pm 0.73^{\mathrm{Ca}}$ & $21.56 \pm 1.07^{\mathrm{Ab}}$ & $14.42 \pm 0.24^{\mathrm{Ca}}$ & $17.02 \pm 0.64^{\mathrm{BCa}}$ \\
\hline Pseudomonas aeroginosa & $13.52 \pm 0.82^{\mathrm{Abc}}$ & $9.13 \pm 0.30^{\mathrm{BCb}}$ & $13.80 \pm 0.38^{\mathrm{Ad}}$ & $8.49 \pm 0.04^{\mathrm{Cf}}$ & $10.28 \pm 0.37^{\mathrm{Bcd}}$ \\
\hline Escherichia coli & $11.67 \pm 0.83^{\text {Acde }}$ & $11.04 \pm 0.35^{\mathrm{ABb}}$ & $11.86 \pm 0.28^{\text {Aef }}$ & $9.16 \pm 0.63^{\text {Bde }}$ & $10.33 \pm 0.60^{\mathrm{ABcd}}$ \\
\hline
\end{tabular}

a-f $(\downarrow)$ : Values with the same capital letters in the same column for each analysis differ significantly $(\mathrm{P}<0.05)$.

A-D $(\rightarrow)$ : Values with the same capital letters in the same rows for each analysis differ significantly $(\mathrm{P}<0.05)$.

Table 3

Antibacterial Effects of Different Extracts of Oenothera biennis and Origanum minutiflorum O. Schwarz et. P.

\begin{tabular}{|c|c|c|c|c|c|c|c|c|c|c|}
\hline \multirow{3}{*}{ Species of Bacteria } & \multicolumn{10}{|c|}{ Solvent } \\
\hline & \multicolumn{2}{|c|}{ Ethanol } & \multicolumn{2}{|c|}{ Methanol } & \multicolumn{2}{|c|}{ Diethyl Ether } & \multicolumn{2}{|c|}{ Acetone } & \multicolumn{2}{|c|}{ Chloroform } \\
\hline & $\mathrm{Ob}$ & $\mathrm{Om}$ & $\mathrm{Ob}$ & $\mathrm{Om}$ & $O b$ & $\mathrm{Om}$ & $O b$ & $O m$ & $O b$ & $\mathrm{Om}$ \\
\hline Staphylococcus aureus & ++ & ++ & ++ & + & +++ & ++ & + & + & ++ & + \\
\hline Yersinia enterecolitica & ++ & + & + & + & +++ & ++ & ++ & + & ++ & + \\
\hline Salmonella Typhimurium & +++ & ++ & ++ & + & +++ & ++ & ++ & + & ++ & ++ \\
\hline Listeria monocytogenes & +++ & +++ & ++ & ++ & +++ & +++ & ++ & ++ & ++ & ++ \\
\hline Enterococcus feacalis & ++ & + & ++ & + & +++ & + & + & - & + & + \\
\hline Enterobacter aerogenes & ++ & + & + & + & ++ & + & + & + & + & + \\
\hline Shigella flexneri & ++ & + & ++ & + & + & ++ & + & + & ++ & + \\
\hline Bacillus cereus & +++ & +++ & +++ & ++ & +++ & +++ & ++ & + & +++ & ++ \\
\hline Pseudomonas aeroginosa & ++ & ++ & - & + & +++ & ++ & - & - & + & + \\
\hline Escherichia coli & ++ & + & + & + & +++ & + & ++ & + & + & + \\
\hline
\end{tabular}

7-9 mm zone diameter: -, 9-12 mm zone diameter: +,12-18 mm zone diameter :++, >18 mm zone diameter:+++, Ob: Oenothera biennis, Om: Origanum minutiflorum O. Schwarz et. P. H. Davis 
Akarca and Tomar / Selcuk J Agr Food Sci, (2020) 34 (1), 78-83

Table 4

MIC (mg/L) and MBC (mg/mL) Values of Different Extracts of Oenothera biennis.

\begin{tabular}{|c|c|c|c|c|c|c|c|c|c|c|}
\hline \multirow{3}{*}{$\begin{array}{l}\text { Species } \\
\text { of Bacte- } \\
\text { ria }\end{array}$} & \multicolumn{10}{|c|}{ Solvent } \\
\hline & \multicolumn{2}{|c|}{ Ethanol } & \multicolumn{2}{|c|}{ Methanol } & \multicolumn{2}{|c|}{ Diethyl Ether } & \multicolumn{2}{|c|}{ Acetone } & \multicolumn{2}{|c|}{ Chloroform } \\
\hline & MIC & $\mathrm{MBC}$ & MIC & $\mathrm{MBC}$ & MIC & MBC & MIC & $\mathrm{MBC}$ & MIC & $\mathrm{MBC}$ \\
\hline $\begin{array}{l}\text { Staphylo- } \\
\text { coccus } \\
\text { aureus }\end{array}$ & $\begin{array}{c}0.070 \pm 0 \\
023^{\mathrm{Bab}}\end{array}$ & $\begin{array}{c}11.72 \pm 3.9 \\
1^{\mathrm{Aa}}\end{array}$ & $\begin{array}{c}0.093 \pm 0.0 \\
00^{\mathrm{Bab}}\end{array}$ & $\underset{\mathrm{Ac}}{15.63 \pm 0.00}$ & $\begin{array}{c}0.035 \pm 0.0 \\
12^{\mathrm{Ba}}\end{array}$ & $\begin{array}{c}11.72 \pm 3 \\
91^{\mathrm{Ab}}\end{array}$ & $\begin{array}{c}0.562 \pm 0 . \\
188^{\text {Aab }}\end{array}$ & $\begin{array}{c}156.25 \pm 9 \\
3.75^{\mathrm{Ab}}\end{array}$ & $\begin{array}{c}0.139 \pm 0.0 \\
45^{\mathrm{Bab}}\end{array}$ & $\begin{array}{c}39.07 \pm 23 \\
44^{\text {Acd }}\end{array}$ \\
\hline $\begin{array}{l}\text { Yersinia } \\
\text { entereco- } \\
\text { litica }\end{array}$ & $\begin{array}{c}0.046 \pm 0 \\
000^{\mathrm{Bb}}\end{array}$ & $\begin{array}{c}15.63 \pm 0.0 \\
0^{\mathrm{Ba}}\end{array}$ & $\begin{array}{c}0.281 \pm 0.0 \\
94^{\mathrm{Aab}}\end{array}$ & $\underset{\mathrm{Ac}}{62.50 \pm 0.00}$ & $\begin{array}{c}0.070 \pm 0.0 \\
24^{\mathrm{ABa}}\end{array}$ & $\begin{array}{c}11.72 \pm 9 \\
91^{\mathrm{Bb}}\end{array}$ & $\begin{array}{c}0.281 \pm 0 . \\
094^{\mathrm{Abc}}\end{array}$ & $\begin{array}{c}39.07 \pm 23 \\
44^{\mathrm{ABb}}\end{array}$ & $\begin{array}{c}0.093 \pm 0.0 \\
00^{\mathrm{ABab}}\end{array}$ & $\begin{array}{c}23.44 \pm 7.8 \\
1^{\mathrm{ABcd}}\end{array}$ \\
\hline $\begin{array}{l}\text { Salmonel- } \\
\text { la Typhi- } \\
\text { murium }\end{array}$ & $\begin{array}{c}0.035 \pm 0 \\
012^{\text {Aab }}\end{array}$ & $\begin{array}{c}11.72 \pm 3.9 \\
1^{\mathrm{Aa}}\end{array}$ & $\begin{array}{c}0.234 \pm 0.1 \\
41^{\mathrm{Aab}}\end{array}$ & $\begin{array}{c}23.44 \pm 7.81 \\
\mathrm{Ac}\end{array}$ & $\begin{array}{c}0.023 \pm 0.0 \\
00^{\mathrm{Aa}}\end{array}$ & $\begin{array}{c}19.53 \pm 11 \\
.72^{\mathrm{Ab}}\end{array}$ & $\begin{array}{c}0.187 \pm 0 . \\
000^{\mathrm{Ac}}\end{array}$ & $\begin{array}{c}31.25 \pm 0.0 \\
0^{\mathrm{Ab}}\end{array}$ & $\begin{array}{c}0.281 \pm 0.0 \\
94^{\mathrm{Aab}}\end{array}$ & $\begin{array}{c}23.44 \pm 7.8 \\
1^{\text {Acd }}\end{array}$ \\
\hline $\begin{array}{l}\text { Listeria } \\
\text { monocy- } \\
\text { togenes }\end{array}$ & $\begin{array}{c}0.017 \pm 0 \\
06^{\mathrm{Ab}}\end{array}$ & $\begin{array}{c}15.63 \pm 0.0 \\
0^{\mathrm{Aa}}\end{array}$ & $\begin{array}{c}0.070 \pm 0.0 \\
24^{\mathrm{Ab}}\end{array}$ & $\begin{array}{c}19.53 \pm 11.7 \\
2^{\mathrm{Ac}}\end{array}$ & $\begin{array}{c}0.017 \pm 0.0 \\
06^{\mathrm{Aa}}\end{array}$ & $\begin{array}{c}7.81 \pm 0.0 \\
0^{\mathrm{Ab}}\end{array}$ & $\begin{array}{c}0.117 \pm 0 . \\
071^{\mathrm{Ac}}\end{array}$ & $\begin{array}{c}23.44 \pm 7.8 \\
1^{\mathrm{Ab}}\end{array}$ & $\begin{array}{c}0.035 \pm 0.0 \\
12^{\mathrm{Ab}}\end{array}$ & $\begin{array}{c}15.63 \pm 0.0 \\
0^{\mathrm{Ad}}\end{array}$ \\
\hline $\begin{array}{l}\text { Entero- } \\
\text { coccus } \\
\text { feacalis }\end{array}$ & $\begin{array}{c}0.035 \pm 0 \\
012^{\mathrm{Bab}}\end{array}$ & $\begin{array}{c}11.72 \pm 3.9 \\
1^{\mathrm{Aa}}\end{array}$ & $\begin{array}{c}0.140 \pm 0.0 \\
47^{\mathrm{Bab}}\end{array}$ & $\underset{\mathrm{Ac}}{62.50 \pm 0.00}$ & $\begin{array}{c}0.029 \pm 0.0 \\
18^{\mathrm{Ba}}\end{array}$ & $\begin{array}{c}39.07 \pm 23 \\
.44^{\mathrm{Ab}}\end{array}$ & $\begin{array}{c}0.562 \pm 0 . \\
188^{\mathrm{Aab}}\end{array}$ & $\begin{array}{c}312.50 \pm 1 \\
87.5^{\mathrm{Aa}}\end{array}$ & $\begin{array}{c}0.140 \pm 0.0 \\
47^{\mathrm{Bab}}\end{array}$ & $\begin{array}{c}187.50 \pm 62 \\
.5^{\mathrm{Ab}}\end{array}$ \\
\hline $\begin{array}{l}\text { Entero- } \\
\text { bacter } \\
\text { aerogenes }\end{array}$ & $\begin{array}{c}0.070 \pm 0 \\
024^{\mathrm{Bab}}\end{array}$ & $\begin{array}{c}15.63 \pm 0.0 \\
0^{\mathrm{Aa}}\end{array}$ & $\begin{array}{c}0.281 \pm 0.0 \\
94^{\mathrm{ABab}}\end{array}$ & $\begin{array}{c}93.75 \pm 32.2 \\
5^{\mathrm{Abc}}\end{array}$ & $\begin{array}{c}0.070 \pm 0.0 \\
24^{\mathrm{Ba}}\end{array}$ & $\begin{array}{c}62.50 \pm 0 \\
00^{\mathrm{Aab}}\end{array}$ & $\begin{array}{c}0.374 \pm 0 \\
000^{\mathrm{Abc}}\end{array}$ & $\begin{array}{c}312.50 \pm 1 \\
87.5^{\mathrm{Aa}}\end{array}$ & $\begin{array}{c}0.105 \pm 0.0 \\
82^{\mathrm{Bab}}\end{array}$ & $\begin{array}{c}156.25 \pm 93 \\
.75^{\mathrm{Abc}}\end{array}$ \\
\hline $\begin{array}{l}\text { Shigella } \\
\text { flexneri }\end{array}$ & $\begin{array}{c}0.046 \pm 0 \\
000^{\mathrm{Ab}}\end{array}$ & $\begin{array}{c}93.75 \pm 31 . \\
25^{\mathrm{Aa}}\end{array}$ & $\begin{array}{c}0.234 \pm 0.1 \\
41^{\text {Aab }}\end{array}$ & $\begin{array}{c}125.00 \pm 0.0 \\
0^{\mathrm{Abc}}\end{array}$ & $\begin{array}{c}0.035 \pm 0.0 \\
12^{\mathrm{Aa}}\end{array}$ & $\begin{array}{c}46.88 \pm 15 \\
.63^{\text {Aab }}\end{array}$ & $\begin{array}{c}0.140 \pm 0 . \\
047^{\mathrm{Ac}}\end{array}$ & $\begin{array}{c}187.50 \pm 6 \\
2.5^{\mathrm{A}} \mathrm{b}\end{array}$ & $\begin{array}{c}0.070 \pm 0.0 \\
24^{\mathrm{Ab}}\end{array}$ & $\begin{array}{c}78.13 \pm 46 . \\
87^{\text {Abcd }}\end{array}$ \\
\hline $\begin{array}{l}\text { Bacillus } \\
\text { cereus } \\
\text { Pseudo- }\end{array}$ & $\begin{array}{c}0.017 \pm 0 \\
006^{\mathrm{Ab}}\end{array}$ & $\begin{array}{c}11.72 \pm 0.0 \\
0^{\mathrm{Aa}}\end{array}$ & $\begin{array}{c}0.035 \pm 0.0 \\
12^{\mathrm{Ab}}\end{array}$ & $\begin{array}{c}20.03 \pm 12.2 \\
2^{\mathrm{Ac}}\end{array}$ & $\begin{array}{c}0.011 \pm 0.0 \\
00^{\mathrm{Aa}}\end{array}$ & $\begin{array}{c}15.63 \pm 0 \\
00^{\mathrm{Ab}}\end{array}$ & $\begin{array}{c}0.093 \pm 0 . \\
000^{\mathrm{Ac}}\end{array}$ & $\begin{array}{c}15.63 \pm 0.0 \\
0^{\mathrm{Ab}}\end{array}$ & $\begin{array}{c}0.138 \pm 0.0 \\
92^{\mathrm{Aab}}\end{array}$ & $\begin{array}{c}11.72 \pm 3.9 \\
1^{\mathrm{Ad}}\end{array}$ \\
\hline $\begin{array}{l}\text { monas } \\
\text { aerogino- } \\
\text { sa }\end{array}$ & $\begin{array}{c}0.140 \pm 0 . \\
047^{\mathrm{Ba}}\end{array}$ & $\begin{array}{c}132.82 \pm 11 \\
7.18^{\mathrm{Ba}}\end{array}$ & $\begin{array}{c}0.374 \pm 0.0 \\
00^{\mathrm{ABa}}\end{array}$ & $\begin{array}{c}375.00 \pm 12 \\
5.00^{\mathrm{ABa}}\end{array}$ & $\begin{array}{c}0.035 \pm 0.0 \\
12^{\mathrm{Ba}}\end{array}$ & $\begin{array}{c}93.75 \pm 31 \\
.25^{\mathrm{Ba}}\end{array}$ & $\begin{array}{c}0.750 \pm 0 . \\
000^{\mathrm{Aa}}\end{array}$ & $>500.00$ & $\begin{array}{c}0.469 \pm 0.2 \\
82^{\mathrm{ABa}}\end{array}$ & $\begin{array}{c}500.00 \pm 0 . \\
00^{\mathrm{Aa}}\end{array}$ \\
\hline $\begin{array}{l}\text { Escheric- } \\
\text { hia coli }\end{array}$ & $\begin{array}{c}0.117 \pm 0 \\
071^{\text {Aab }}\end{array}$ & $\begin{array}{c}93.75 \pm 31 . \\
25^{\mathrm{Aa}}\end{array}$ & $\begin{array}{c}0.281 \pm 0.0 \\
94^{\mathrm{Aab}}\end{array}$ & $\begin{array}{c}312.50 \pm 18 \\
7.50^{\mathrm{Aab}}\end{array}$ & $\begin{array}{c}0.058 \pm 0.0 \\
35^{\mathrm{Aa}}\end{array}$ & $\begin{array}{c}39.07 \pm 23 \\
.44^{\mathrm{Ab}}\end{array}$ & $\begin{array}{c}0.058 \pm 0 . \\
035^{\mathrm{Ac}}\end{array}$ & $\begin{array}{c}46.88 \pm 15 \\
62^{\mathrm{Ab}}\end{array}$ & $\begin{array}{c}0.105 \pm 0.0 \\
82^{\text {Aab }}\end{array}$ & $\begin{array}{c}125.00 \pm 0 . \\
00^{\mathrm{Abcd}}\end{array}$ \\
\hline
\end{tabular}

Table 5

MIC (mg/L) and MBC (mg/mL) Values of Different Extracts Origanum minutiflorum O. Schwarz et. P. H.

\begin{tabular}{|c|c|c|c|c|c|c|c|c|c|c|}
\hline \multirow{3}{*}{$\begin{array}{l}\text { Species } \\
\text { of Bacte- } \\
\text { ria }\end{array}$} & \multicolumn{10}{|c|}{ Solvent } \\
\hline & \multicolumn{2}{|c|}{ Ethanol } & \multicolumn{2}{|c|}{ Methanol } & \multicolumn{2}{|c|}{ Diethyl Ether } & \multicolumn{2}{|c|}{ Acetone } & \multicolumn{2}{|c|}{ Chloroform } \\
\hline & MIC & $\mathrm{MBC}$ & MIC & $\mathrm{MBC}$ & MIC & $\mathrm{MBC}$ & MIC & $\mathrm{MBC}$ & $\mathrm{MIC}$ & $\mathrm{MBC}$ \\
\hline $\begin{array}{l}\text { Staphylo- } \\
\text { coccus } \\
\text { aureus }\end{array}$ & $\begin{array}{c}0.140 \pm 0.0 \\
47^{\mathrm{ABa}}\end{array}$ & $\begin{array}{c}93.75 \pm 31 . \\
25^{\text {Aab }}\end{array}$ & $\begin{array}{c}0.187 \pm 0 \\
000^{\mathrm{Aba}}\end{array}$ & $\begin{array}{c}187.50 \pm 62 \\
.50^{\mathrm{Aab}}\end{array}$ & $\begin{array}{c}0.093 \pm 0 . \\
000^{\mathrm{Bab}}\end{array}$ & $\begin{array}{c}78.13 \pm 46 . \\
87^{\mathrm{Aa}}\end{array}$ & $\begin{array}{c}0.281 \pm 0 . \\
094^{\mathrm{Abc}}\end{array}$ & $\begin{array}{c}140.63 \pm 10 \\
9.38^{\mathrm{Aab}}\end{array}$ & $\begin{array}{c}0.187 \pm 0 . \\
000^{\mathrm{Aba}}\end{array}$ & $\begin{array}{c}62.50 \pm 0.0 \\
00^{\mathrm{Abc}}\end{array}$ \\
\hline $\begin{array}{l}\text { Yersinia } \\
\text { entereco- } \\
\text { litica }\end{array}$ & $\begin{array}{c}0.187 \pm 0.0 \\
00^{\mathrm{Aa}}\end{array}$ & $\begin{array}{c}78.13 \pm 46 . \\
87^{\mathrm{ABab}}\end{array}$ & $\begin{array}{c}0.234 \pm 0 \\
140^{\mathrm{Aa}}\end{array}$ & $\begin{array}{c}125.00 \pm 0 \\
00 \mathrm{~A}^{\mathrm{Bab}}\end{array}$ & $\begin{array}{c}0.140 \pm 0 . \\
047^{\mathrm{Aab}}\end{array}$ & $\begin{array}{c}46.88 \pm 15 \\
63^{\mathrm{Ba}}\end{array}$ & $\begin{array}{c}0.374 \pm 0 \\
000^{\mathrm{Abc}}\end{array}$ & $\begin{array}{c}187.50 \pm 62 \\
50^{\mathrm{Aab}}\end{array}$ & $\begin{array}{c}0.281 \pm 0 \\
094^{\mathrm{Aa}}\end{array}$ & $\begin{array}{c}125.00 \pm 0 . \\
00^{\mathrm{ABbc}}\end{array}$ \\
\hline $\begin{array}{l}\text { Salmonel- } \\
\text { la Typhi- } \\
\text { murium }\end{array}$ & $\begin{array}{c}0.117 \pm 0.0 \\
71^{\mathrm{Aa}}\end{array}$ & $\begin{array}{c}93.75 \pm 31 . \\
25^{\text {Aab }}\end{array}$ & $\begin{array}{c}0.281 \pm 0 \\
094^{\mathrm{Aa}}\end{array}$ & $\begin{array}{c}156.25 \pm 93 \\
.75^{\mathrm{Aab}}\end{array}$ & $\begin{array}{c}0.093 \pm 0 \\
000^{\mathrm{Aab}}\end{array}$ & $\begin{array}{c}78.13 \pm 46 . \\
87^{\mathrm{Aa}}\end{array}$ & $\begin{array}{c}0.187 \pm 0 . \\
000^{\mathrm{Abc}}\end{array}$ & $\begin{array}{c}125.00 \pm 0.0 \\
0^{\text {Aab }}\end{array}$ & $\begin{array}{c}0.140 \pm 0 . \\
047^{\mathrm{Aa}}\end{array}$ & $\begin{array}{c}93.75 \pm 31 . \\
25^{\mathrm{Abc}}\end{array}$ \\
\hline $\begin{array}{l}\text { Listeria } \\
\text { monocy- } \\
\text { togenes }\end{array}$ & $\begin{array}{c}0.046 \pm 0.0 \\
00^{\mathrm{ABa}}\end{array}$ & $\begin{array}{c}19.53 \pm 11 \\
72^{\mathrm{Ab}}\end{array}$ & $\begin{array}{c}0.070 \pm 0 \\
024^{\mathrm{Aba}}\end{array}$ & $\begin{array}{c}46.88 \pm 15 \\
63^{\mathrm{Ab}}\end{array}$ & $\begin{array}{c}0.029 \pm 0 \\
018^{\mathrm{Bb}}\end{array}$ & $\begin{array}{c}11.72 \pm 3.9 \\
1^{\mathrm{Aa}}\end{array}$ & $\begin{array}{c}0.093 \pm 0 . \\
000^{\mathrm{Ac}}\end{array}$ & $\begin{array}{c}39.07 \pm 23.4 \\
4^{\mathrm{Ab}}\end{array}$ & $\begin{array}{c}0.070 \pm 0 \\
018^{\mathrm{Aba}}\end{array}$ & $\begin{array}{c}31.25 \pm 0.0 \\
0^{\mathrm{Ac}}\end{array}$ \\
\hline $\begin{array}{l}\text { Entero- } \\
\text { coccus } \\
\text { feacalis }\end{array}$ & $\begin{array}{c}0.234 \pm 0.1 \\
41^{\mathrm{Aa}}\end{array}$ & $\begin{array}{c}125.00 \pm 0 \\
00^{\mathrm{Aab}}\end{array}$ & $\begin{array}{c}0.422 \pm 0 \\
328^{\mathrm{Aa}}\end{array}$ & $\begin{array}{c}312.50 \pm 18 \\
7.5^{\mathrm{Aa}}\end{array}$ & $\begin{array}{c}0.140 \pm 0 . \\
047^{\mathrm{Aab}}\end{array}$ & $\begin{array}{c}93.75 \pm 31 \\
25^{\mathrm{Aa}}\end{array}$ & $\begin{array}{c}0.469 \pm 0 . \\
282^{\text {Aab }}\end{array}$ & $>500.00$ & $\begin{array}{c}0.187 \pm 0 \\
000^{\mathrm{Aa}}\end{array}$ & $\begin{array}{c}187.50 \pm 62 \\
.5^{\mathrm{Abc}}\end{array}$ \\
\hline $\begin{array}{l}\text { Entero- } \\
\text { bacter } \\
\text { aeroge- } \\
\text { nes }\end{array}$ & $\begin{array}{c}0.140 \pm 0.0 \\
47^{\mathrm{Aa}}\end{array}$ & $\begin{array}{c}187.50 \pm 6 \\
2.5^{\mathrm{ABa}}\end{array}$ & $\begin{array}{c}0.187 \pm 0 \\
000^{\mathrm{Aa}}\end{array}$ & $\begin{array}{c}250.00 \pm 0 \\
00^{\mathrm{ABab}}\end{array}$ & $\begin{array}{c}0.234 \pm 0 \\
147^{\mathrm{Aa}}\end{array}$ & $\begin{array}{c}125.00 \pm 0 . \\
00^{\mathrm{Ba}}\end{array}$ & $\begin{array}{c}0.140 \pm 0 . \\
047^{\mathrm{Abc}}\end{array}$ & $\begin{array}{c}375.00 \pm 12 \\
5.0^{\text {Aab }}\end{array}$ & $\begin{array}{c}0.281 \pm 0 \\
094^{\mathrm{Aa}}\end{array}$ & $\begin{array}{c}250.00 \pm 0 . \\
00^{\mathrm{ABab}}\end{array}$ \\
\hline $\begin{array}{l}\text { Shigella } \\
\text { flexneri }\end{array}$ & $\begin{array}{c}0.187 \pm 0.0 \\
00^{\mathrm{Aa}}\end{array}$ & $\begin{array}{c}156.25 \pm 9 \\
3.75^{\mathrm{Bab}}\end{array}$ & $\begin{array}{c}0.281 \pm 0 \\
094^{\mathrm{Aa}}\end{array}$ & $\begin{array}{c}125.00 \pm 0 . \\
00^{\mathrm{Bab}}\end{array}$ & $\begin{array}{c}0.117 \pm 0 . \\
071^{\text {Aab }}\end{array}$ & $\begin{array}{c}156.25 \pm 9 \\
3.75^{\mathrm{Ba}}\end{array}$ & $\begin{array}{c}0.234 \pm 0 . \\
141^{\mathrm{Abc}}\end{array}$ & $\begin{array}{c}500.00 \pm 0.0 \\
0^{\text {Aab }}\end{array}$ & $\begin{array}{c}0.187 \pm 0 . \\
000^{\mathrm{Aa}}\end{array}$ & $\begin{array}{c}375.00 \pm 12 \\
5.0^{\mathrm{ABa}}\end{array}$ \\
\hline $\begin{array}{l}\text { Bacillus } \\
\text { cereus } \\
\text { Pseudo- }\end{array}$ & $\begin{array}{c}0.078 \pm 0.3 \\
2^{\mathrm{Aa}}\end{array}$ & $\begin{array}{c}39.07 \pm 23 \\
44^{\mathrm{Aab}}\end{array}$ & $\begin{array}{c}0.117 \pm 0 \\
071^{\mathrm{Aa}}\end{array}$ & $\begin{array}{c}46.88 \pm 15 \\
63^{\mathrm{Ab}}\end{array}$ & $\begin{array}{c}0.029 \pm 0 \\
018^{\mathrm{Ab}}\end{array}$ & $\begin{array}{c}7.81 \pm 0.00 \\
\text { Aa }\end{array}$ & $\begin{array}{c}0.058 \pm 0 \\
035^{\mathrm{Ac}}\end{array}$ & $\begin{array}{c}78.13 \pm 46.8 \\
8^{\mathrm{Ab}}\end{array}$ & $\begin{array}{c}0.117 \pm .0 . \\
071^{\mathrm{Aa}}\end{array}$ & $\begin{array}{c}19.53 \pm 11 \\
72^{\mathrm{Ac}}\end{array}$ \\
\hline $\begin{array}{l}\text { monas } \\
\text { aerogino- } \\
\text { sa }\end{array}$ & $\begin{array}{c}0.070 \pm 0.0 \\
24^{\mathrm{Ba}}\end{array}$ & $\begin{array}{c}93.75 \pm 31 \\
25^{\mathrm{Bab}}\end{array}$ & $\begin{array}{c}0.234 \pm 0 . \\
141^{\mathrm{Ba}}\end{array}$ & $\begin{array}{c}250.00 \pm 0 \\
00^{\mathrm{Bab}}\end{array}$ & $\begin{array}{c}0.070 \pm 0 . \\
024^{\mathrm{Bab}}\end{array}$ & $\begin{array}{c}156.25 \pm 9 \\
3.75^{\mathrm{Ba}}\end{array}$ & $\begin{array}{c}0.750 \pm 0 . \\
000^{\mathrm{Aa}}\end{array}$ & $\begin{array}{c}500.00 \pm 0.0 \\
0^{\mathrm{Aa}}\end{array}$ & $\begin{array}{c}0.281 \pm 0 . \\
094^{\mathrm{Ba}}\end{array}$ & $\begin{array}{c}156.25 \pm 93 \\
.75^{\mathrm{Bbc}}\end{array}$ \\
\hline $\begin{array}{l}\text { Escheric- } \\
\text { hia coli }\end{array}$ & $\begin{array}{c}0.140 \pm 0.0 \\
47^{\text {Aa }}\end{array}$ & $\begin{array}{c}62.50 \pm 0.0 \\
0^{\text {Aab }}\end{array}$ & $\begin{array}{c}0.187 \pm 0 \\
000^{\mathrm{Aa}}\end{array}$ & $\begin{array}{c}93.75 \pm 31 . \\
25^{\text {Aab }}\end{array}$ & $\begin{array}{c}0.140 \pm 0 \\
047^{\text {Aab }}\end{array}$ & $\begin{array}{c}125.00 \pm 0 . \\
00^{\mathrm{Aa}}\end{array}$ & $\begin{array}{c}0.187 \pm 0 . \\
000^{\mathrm{Abc}}\end{array}$ & $\begin{array}{c}312.50 \pm 18 \\
7.5^{\text {Aab }}\end{array}$ & $\begin{array}{c}0.140 \pm 0 . \\
047^{\mathrm{Aa}}\end{array}$ & $\begin{array}{c}93.75 \pm 31 \\
25^{\mathrm{Abc}}\end{array}$ \\
\hline
\end{tabular}


The highest antibacterial effect was observed in the diethyl ether extract on Listeria monocytogenes with a zone diameter of $23.98 \mathrm{~mm}$ whereas the lowest antibacterial effect was on Enterococcus faecalis with a zone diameter of $8.13 \mathrm{~mm}$ in the acetone extract $(\mathrm{P}<0.05)$

Among the five different extracts, the highest antibacterial effect was determined in the extracts prepared with diethyl ether (Table 3), followed by ethanol and chloroform extracts $(\mathrm{P}<0.05)$. Among the extracts, the lowest antibacterial effect on ten different pathogenic bacterial strains was determined in the acetone extract $(\mathrm{P}<0.05)$. As a result of similar studies on the subject, it has been stated that the high antibacterial effects of extracts were caused by carvacrol and thymol, which are abundant in the structure of plants (Aslim and Yucel, 2008; Bostancioglu et al., 2012).

Of the five different extracts obtained from Oenothera biennis, the lowest MIC and MBC values were determined in diethyl ether, ethanol and methanol extracts, respectively, whereas the highest MIC and MBC values were determined in the acetone extract (Table 4; $\mathrm{P}<0.05)$. The lowest MIC values of the extracts were on Bacillus cereus and Listeria monocytogenes, respectively, whereas the highest values were on Pseudomonas aeroginosa, Yersinia enterecolitica and Enterobacter aerogenes, respectively $(\mathrm{P}<0.05)$.

The lowest MIC and MBC values of the plant extracts on ten different pathogenic bacteria strains were $0.011 \mathrm{mg} / \mathrm{L}$ and $7.81 \mathrm{mg} / \mathrm{mL}$ in the diethyl ether extract against Bacillus cereus, whereas the highest values were $0.750 \mathrm{mg} / \mathrm{L}$ and $>500 \mathrm{mg} / \mathrm{mL}$ in the acetone extract against Pseudomonas aeroginosa (Table 4).

It was determined that the lowest MIC and MBC values, as in Oenothera biennis, were determined in the Origanum minutiflorum O. Schwarz et. P. H. extract obtained from the diethyl ether extract whereas the highest MIC and MBC values were determined in the acetone extract (Table 5; $\mathrm{P}<0.05$ ). The lowest MIC value of the diethyl extract was $0.029 \mathrm{mg} / \mathrm{L}$ on Listeria

Table 6

Analysis Results of Variance Analysis of Oenothera biennis and Origanum minutiflorum O. Schwarz et. P. H. Davis on Solvent, Antibacterial Effect, MIC and MBC Values (P value)

\begin{tabular}{lccc}
\hline Factors & Antibacterial Effect & MIC & MBC \\
\hline Plant species & $<0.0001$ & 0.057 & $<0.0001$ \\
Bacteria species & $<0.0001$ & $<0.0001$ & $<0.0001$ \\
Solvent type & $<0.0001$ & $<0.0001$ & $<0.0001$ \\
Solvent type x Bacteria species & $<0.0001$ & $<0.0001$ & 0.05 \\
Solvent type x Plant species & $<0.0001$ & 0.207 & 0.204 \\
Bacteria species x Plant species & $<0.0001$ & 0.360 & 0.01 \\
Solvent type x Bacteria species x Plant species & $<0.0001$ & 0.809 & 0.136 \\
\hline
\end{tabular}

$\mathrm{P}<0.05$ : Statistically significant, $\mathrm{P}<0.01$ : Statistically very significant, $\mathrm{P}<0.0001$ : Statistically too much significant, $\mathrm{P}>0.05:$ Not statistically significant.

\section{Conclusion}

According to the results of this study, both plant species, especially Oenothera biennis, were found to have high antibacterial effects. The fact that the highest monocytogenes and Bacillus cereus, while the lowest MBC value of the diethyl extract was $7.81 \mathrm{mg} / \mathrm{mL}$ on Bacillus cereus $(\mathrm{P}<0.05)$. The acetone extract of the Origanum minutiflorum O. Schwarz et. P. H. Davis had the highest MIC and MBC values against ten different food-borne pathogenic bacteria among the extracts obtained with five different solvents $(\mathrm{P}<0.05)$. The highest MIC and MBC values of this extract were found to be $0.750 \mathrm{mg} / \mathrm{L}$ and $>500 \mathrm{mg} / \mathrm{mL}$ on Pseudomonas aeroginosa $(\mathrm{P}<0.05)$.

According to variance analysis results, the antibacterial effect of extracts obtained from two different plants using five different solvents on ten different foodborne pathogens, it was found that plant species, bacterial species, solvent species, solvent species $\mathrm{x}$ bacteria species interaction, solvent species $\mathrm{x}$ plant species interaction, bacterial species $\mathrm{x}$ plant species interaction and solvent species $\mathrm{x}$ bacterial species $\mathrm{x}$ plant species interaction were found to be significant (P<0.0001; Table 6).

Similarly, bacterial species, solvent type, solvent type $\mathrm{x}$ bacterial species interactions had a significant effect on the MIC value $(\mathrm{P}<0.0001)$. In terms of the effect on the MBC value, it was determined that plant species, bacterial species, solvent type interactions were significant at $\mathrm{P}<0.0001$, while solvent type $\mathrm{x}$ bacterial interaction was significant at $\mathrm{P}<0.05$ and bacterial species $\mathrm{x}$ plant species interaction was significant at $\mathrm{P}<0.01$ (Table 6).

Albayrak and Aksoy (2017), similar to our study, have stated that the antibacterial effect of Origanum minutiflorum was high. As a result of the research, the highest antibacterial effect of ethanol extract has been reported to be on Aeromonas hydrophilic with a 24mm-zone diameter and Streptococcus pneumonia with an 18-mm-zone diameter, respectively. The researchers have reported that the lowest MIC and MBC values of ethanol extract of the plant were $0.78 \mathrm{mg} / \mathrm{mL}$ and 0.78 $\mathrm{mg} / \mathrm{mL}$, respectively, on Klebsiella pneumonia. activity was found in diethyl ether extract among different solvents used showed that the components found in the composition of the leaves of the plants which exhibit antibacterial effect were best decomposed in this solvent. 
In recent years, the trend towards the use of natural products as an alternative to artificial food additives and pharmaceuticals has led manufacturers to research this subject. Successful results obtained from many studies show the usability of such products that are obtained from a large number of plants.

The cultivation of these two plants, which have been consumed for different purposes for many years, in larger areas and the products such as extracts, essential oils, and essences to be obtained from these plants can be used as natural preservatives, shelf-life extenders and antibacterials in food industry, and medicine industry including pharmacology, medical and veterinary.

\section{References}

Akarca G (2019). Composition and antibacterial effect on food borne pathogens of Hibiscus surrattensis L. calyces essential oil. Industrial Crops \& Products 137: 285-289.

Albayrak S, Aksoy A (2017). Phenolic contents and biological activity of endemic Origanum minutiflorum grown in Turkey. Indian Journal of Pharmaceutical Education and Research 53(1): 160-170.

Anonymous (2018). Eucast, European Commitee on Antimicrobial Susceptibilty Testing, http://www.eucast.org/fileadmin/src/media/PDFs/EUC AST_files/Breakpoint_tables/v_8.0_Breakpoint_Table s.pdf

Arimura T, Kojima-Yuasa A, Watanabe S, Suzuki M, Kennedy DO, Matsui-Yuasa I (2003a). Role of intracellular reactive oxygen species and mitochondrial dysfunction in evening primrose extract-induced apoptosis in Ehrlich ascites tumor cells. ChemicoBiological Interactions 145: 337-347.

Arimura T, Kojima-Yuasa A, Suzuki M, Kennedy DO, Matsui-Yuasa I (2003b). Caspase-independent apoptosis induced byevening primrose extract in Ehrlich ascites tumour cells. Cancer Letters 201: 9-16.

Arimura T, Kojima-Yuasa A, Kennedy DO, Matsui-Yuasa I (2004). Reactive oxygen species-independent G1 arrest induced by evening primrose extract in Ehrlich ascites tumour cells, Cancer Letters 207: 19-25.

Aslım B, Yucel N (2008). In vitro antimicrobial activity of essential oil from endemic Origanum minutiflorum on ciprofloxacin-resistant Campylobacter spp. Food Chemistry 107: 602-606.

Azizi A, Yan F, Honermeier B (2009). Herbage yield, essential oil content and composition of three oregano (Origanum vulgare L.) populations as affected by soil moisture regimes and nitrogen supply. Industrial Crops \& Products 29(2): 554-561.

Bauer AW, Kirby WMM, Sherris JC, Turck M (1966) Antibiotic susceptibility testing by a standardized single disk method. American Journal of Clinical Pathology 36: 493-496.

Baydar H (2005). The effect of different picking times on volatile oil content and volatile oil components in highland thyme (Origanum minutiflorum O. Schwarz et. P. H. Davis). Mediterranean Agricultural sciences 18(2): 175-178.

Becker H (1983). Oil of evening primrose Oenothera biennis, a source of fatty acids of therapeutic and dietectic interest. Z. Phytotherapy 4: 531-536.

Bostancioglu RB, Kurkcuoglu M, Baser KHC, Koparal AT (2012). Assessment of anti-angiogenic and antitumoral potentials of Origanum onites L. essential oil. Food and Chemical Toxicology 50(6): 2002-2008.

By Aamer AA, Abdul-Hafeez MM, Sayed SM (2015). Minimum inhibitory and bactericidal concentrations (MIC \& MBC) of honey and bee propolis against multidrug resistant (MDR) Staphylococcus Spp. isolated from bovine clinical mastitis. Alternative \& Integrative Medicine 3: 171.

Chikezie IO (2017). Determination of minimum inhibitory concentration (MIC) and minimum bactericidal concentration (MBC) using a novel dilution tube method. African Journal of Microbiology Research 11(23): 977-980.

Chishti S, Kaloo ZA, Sultan P (2013). Medicinal importance of genus Origanum: A review. Journal of Pharmacognosy and Phytochemistry 5(10): 170-177.

Cruz-Gálvez AM, Castro-Rosas J, Rodríguez-Marín, ML, Cadena-Ramírez A, Tellez-Jurado A, Tovar-Jiménez $\mathrm{X}$, Chavez-Urbiola EA, Abreu-Corona A, GómezAldapa CA (2018). Antimicrobial activity and physicochemical characterization of a potato starch-based film containing acetonic and methanolic extracts of Hibiscus sabdariffa for use in sausage. LWT Food Science and Technology 93: 300-305.

Dhiman A, Nanda A, Ahmad S, Narasimhan B (2011). In vitro antimicrobial activity of methanolic leaf extract of Psidium guajava L. Journal of Pharmacy and Bioallied Sciences 3(2): 226-229.

Karaboduk K, Karabacak O, Karaboduk H, Tekinay T (2014). Chemical analysis and antimicrobial activities of the Origanum vulgare subsp. hirtum. Journal of Environmental Protection and Ecology 15(3A): 12831292.

Morrison KD, Reekie EG (1995). Pattern of defoliation and its effect on photosynthetic capacity in Oenothera biennis. Journal of Ecology 83: 759-767.

Oke F, Aslim B (2010). Biological potentials and cytotoxicity of various extracts from endemic Origanum minutiflorum O. Schwarz and P.H. Davis. Food and Chemical Toxicology 48(6): 1728-1733.

Ozen F, Ekinci FY, Korachi M (2014). The inhibition of Helicobacter pylori infected cells by Origanum minutiflorum. Industrial Crops \& Products 58: 329-34.

Sarikurkcu C, Zengin G, Oskay M, Uysal S, Ceylan R, Aktumsek A (2015). Composition, antioxidant, antimicrobial and enzyme inhibition activities of two Origanum vulgare subspecies (subsp. vulgare and subsp. hirtum) essential oils. Industrial Crops \& Products 70:178-84.

Shukla YN, Srivastava A, Kumar S, Kumar S (1999). Phytotoxic and antimicrobial constituents of Argyreia speciosa and Oenothera biennis. Journal of Ethnopharmacology 67: 241-245. 